\title{
Experimental and numerical simulation of carbon manganese steel for cyclic plastic behaviour
}

\author{
J. Shit, S. Dhar, S. Acharyya*
}

Department of Mechanical Engineering,, Jadavpur University,Kolkata-700032, INDIA

*Corresponding Author: e-mail:sanjib_acharyya@hotmail.com, skacharyya@mech.jdvu.ac.in

\begin{abstract}
The paper deals with finite element modeling of saturated low cycle fatigue and the cyclic hardening phenomena of the materials Sa333 grade 6 carbon steel and SS316 stainless steel. Von Mises' yield function and Chaboche's kinematic hardening rules have been used. The recall terms of Chaboche's kinematic hardening rule is calibrated with a memory function. The evolution law of the memory function is determined from experimental observations. The material constants are identified and derived from experimental results. The FE simulated results are compared with the experimental results from virgin state to saturation state of the materials.
\end{abstract}

Keywords: cyclic plasticity, kinematic hardening, low cyclic fatigue, dilatation

\section{Introduction}

Inelastic material behavior in closed loading path, repeated number of times is known as cyclic plasticity. The experimental observations show a number of related phenomena. Those are i.) Bauschinger effect, ii.) cyclic hardening/softening, iii.) mean stress relaxation and ratcheting. Some materials also shows S-D ( strength differential) effect (Spitzig et al, 1975). Above all, there is additional hardening due to non proportional loading path. Addressing all these phenomena in constitutive equations becomes complicated and difficult to implement numerically.

Bauschinger effect can be explained as translation of the centre of yield surface which is known as kinematic hardening phenomenon. This is generally modeled by using proper evolution laws of back stress tensor. A simplest choice is a linear kinematic hardening law due to Prager [Prager. W, 1956] . Armstrong and Frederick (1966) introduced a nonlinear law with recall term. Chaboche (1986), Ohno (1982) modified Armstrong -Frederick law to have a better matching with experimental results. In general, Armstrong - Frederick law and also its multi segmented version due to Chaboche and Rousselier can not address cyclic hardening / softening phenomenon. So it can model only saturated state but not the transition process from virgin state to saturated state. Chaboche, Dang Van and Cordier (1979) introduced a strain memory surface which can both translate and expand. Thus, controlling isotropic hardening rate cyclic hardening is explained. Ohno and Kachi (1985) modified the concept to achieve a realistic model. But this model can not address fading memory as the non hardening surface can expand only.

As mentioned by Chaboche and Rousselier (1983), the Armstrong - Frederick law can model cyclic mean stress relaxation qualitatively but the rate of relaxation is over estimated. For multi segmented law of Chaboche - Rousselier the relaxation rate is controlled by adjusting recalling terms.

In general, Von Mises' model considers equal flow stress in tension and compression and the effect of hydrostatic stress on flow stress is neglected. But the effect of hydrostatic stress on flow stress is observed in some materials after a few cycles (Mahnken Rolf. 2001). This produces higher flow stress in compression than that in tension ( S-D effect).

Lastly, additional hardening is observed in all metals due to nonproportionality in loading path. In order to study the whole range of principal strain ratio, Ellyin and Xia (1989) carried out biaxial tension/compression tests, thereby confirming general trends. Under non proportional loading the stabilized response is independent of mean strain as in proportional loading case (Benallal et al 1989 ). The present model is formulated in the same line as in reference (Haupt and Kamlah, 1995 ). 


\section{Mathematical Formulation}

Yield Function: - The yield function used in the present model is a Von Mises' yield function. This is as follows

$\Phi=\frac{3}{2}\left(S_{i j}-X_{i j}\right)\left(S_{i j}-X_{i j}\right)-\sigma_{0}^{2}=0$,

$\sigma_{0}=$ Yield stress of the material, $\mathrm{S}_{\mathrm{ij}}=$ deviatoric part of stress tensor,

$\mathrm{X}_{\mathrm{ij}}=$ back stress tensor, also deviatoric in nature,

\subsection{The Flow Rule:}

The Plastic strain rate, $\dot{\varepsilon}_{i j}^{p}$, follows from the flow rules as

$\dot{\varepsilon}_{i j}^{p}=\lambda \frac{\partial \Phi}{\partial \sigma_{i j}}$

Here, $\lambda$ is a scalar multiplier.

\subsection{Kinematic hardening rule:}

For the evolution law of back stress, we restrict ourselves to the functional structure (Haupt et al, 1987) .

$$
X_{i j}=\int_{0}^{z} k\left(z-z^{\prime}\right) \frac{d}{d z^{\prime}} \varepsilon_{i j}^{p}\left(z^{\prime}\right) d z^{\prime}
$$

Here, the back stress tensor is the integrated value of the linear functional of plastic strain history. The Kernel function, $\mathrm{k}\left(\mathrm{z}-\mathrm{z}^{\prime}\right)$, contains the memory properties of the hardening function.

Here, $\mathrm{z}$ is defined as generalized arc length of plastic strain component. In this context, plastic arc length should be defined. The plastic arc length is defined as the accumulated scalar plastic strain i.e.

$$
s(t)=\int_{0}^{t} \sqrt{\frac{2}{3}}\left(\dot{\varepsilon}_{i j}^{p} \dot{\varepsilon}_{i j}^{p}\right)^{\frac{1}{2}} d t,
$$

With, $\quad \dot{s}=\sqrt{\frac{2}{3}}\left(\dot{\varepsilon}_{i j}^{p} \dot{\varepsilon}_{i j}^{p}\right)^{\frac{1}{2}}$

The generalized plastic arc length ' $z$ ' is a functional of plastic arc length, given as

$$
z=\int_{0}^{s} \hat{b}(p(s)) d s
$$

Here $\mathrm{p}(\mathrm{s})$ is a memory function.

Let us consider a special case, for which, $\hat{b}(p(s))=1$ and also the kernel functional

$k(z)=\sum_{i=1}^{N} c_{i} e^{-b_{i} z}$, Here, $c_{i}$ and $b_{i}$ are non-negative material constant. Since, $\dot{z}=\dot{s}$, equation (3) can be written as, $\bar{X}=\sum_{k=1}^{N} \bar{X}_{k}$, with

$$
\dot{\bar{X}}_{k}=c_{k} \overline{\dot{\varepsilon}}_{p}-b_{k} \dot{s} \bar{X}_{k}, \text { Where } \overline{\dot{\varepsilon}}_{p}=\dot{\varepsilon}_{i j}^{p}
$$

The above equation can be recognized easily as the hardening rule proposed by Chobache and Rousselier (1983). With, N=1, it reduces to classical Armstrong - Frederick equation.

It is mentioned earlier that although, Armstrong - Frederick equation (or Chobache - Rousselier multi segmented version) can simulate Bauschinger effect for saturated state but it cannot model cyclic hardening/softening during transition period. Therefore, $\hat{b}(p(s))=1$ is not a realistic choice. From equation (4), $\dot{z}=\hat{b}(p) \dot{s}$, gives a kinematic hardening law as,

$$
\dot{\bar{X}}_{k}=c_{k} \overline{\dot{\varepsilon}}_{p}-b_{k} \hat{b}(p(s)) \dot{s} \bar{X}_{k}
$$


The functional, $\hat{b}(p(s))$, has the following properties.

i) $\hat{b}(p(s)) \geq 0$, for all values of $\mathrm{p}(\mathrm{s})$ ii) $\hat{b}(0)=0$ for $\mathrm{p}(\mathrm{s})=0$ and iii) $\hat{b}^{\prime}(p(s)) \leq 0$ for all possible values of $\mathrm{p}(\mathrm{s})$. One of the simplest choices of $\hat{b}(p(s))$ is given by Benallal et al (1989). That is,

$\hat{b}(p(s))=\frac{1}{1+a p(s)} \quad$ Here, 'a' is a material constant. This choice of $\hat{b}(p)$ gives a kinematic hardening law as

$$
\dot{\bar{X}}_{k}=c_{k} \overline{\dot{\varepsilon}}_{p}-\frac{b_{k}}{1+a p(s)} \dot{s} \bar{X}_{k}
$$

The evolution of back stress will be complete with the evolution equation of memory function, $\mathrm{p}(\mathrm{s})$. For that we come down to uniaxial tension compression loading.

The back stress tensor $X_{\mathrm{ij}}$ is considered as deviatoric in nature. Here, $\mathrm{X}_{11}=\mathrm{X}_{11}$,

$X_{22}=(-1 / 2) X_{11}, X_{33}=(-1 / 2) X_{11}$ and the other components are zero. The norm of the back stress tensor is $\sqrt{\frac{3}{2}}\left|X_{11}\right|$. We define, $\xi=\frac{3}{2} X_{11}$ and $\varepsilon^{p}=\varepsilon_{11}^{p}$. Thus, the norm of the back stress tensor is written as $\sqrt{\frac{2}{3}}|\xi|$. The above hardening equation (7) with $\mathrm{N}=1$ reduces to

$$
\dot{\xi}=\frac{3}{2} c \dot{\varepsilon}_{p}-\frac{b}{1+a p(s)} \dot{s} \xi
$$

Several research workers $[1,12,13]$ used a functional relation of ' $p$ ' as follows

$$
p=\frac{1}{S_{0}} \int_{o}^{s} e^{-(s-\bar{s}) / S_{0}} \sqrt{\frac{2}{3}}|\xi| d \bar{s}
$$

The above equation culminates from a differential equation

$$
\dot{p}=\frac{\dot{s}}{S_{0}}\left(\sqrt{\frac{2}{3}|\xi|}-p\right), \text { With } \mathrm{p}(0)=0
$$

In this equation, $\mathrm{S}_{0}$ is the memory range. That is to say that beyond $\mathrm{S}_{0}$ the plastic strain memory is erased. From differential equation (10) it is seen that

$$
\sqrt{\frac{2}{3}}|\xi|=p(s)+\frac{d p}{d s} S_{0}
$$

This shows clearly that the back stress depends on current memory $\mathrm{p}(\mathrm{s})$ and its history over a plastic arc of length $\mathrm{S}_{0}$.

\subsection{Stationary value:}

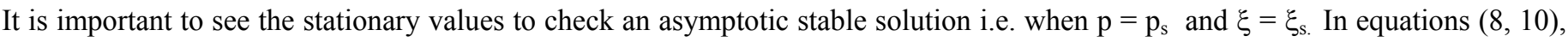
putting $\dot{p}=0$ and $\dot{\xi}=0$ one gets

$$
\left|\xi_{s}\right|=\sqrt{\frac{3}{2}} p_{s}=\frac{\frac{3}{2} \frac{c}{b}}{1-\sqrt{\frac{3}{2}} \frac{c}{b} a}
$$

The solution exists for, $a<\sqrt{\frac{2}{3}} \frac{b}{c}$. Therefore, with this inequality a unique solution can be obtained. The nonnegative constant 'a' controls the rate of stabilization. Here, $\xi_{\mathrm{s}}$ and $\mathrm{p}_{\mathrm{s}}$ are values of $\xi$ and $\mathrm{p}$ at stabilization. 


\section{Material Constants}

Elastic Modulus, E and Poisson's ratio $v$ are obtained from tensile test.

\section{Kinematic hardening variables:}

$\mathrm{C}_{\mathrm{k}}$ and $\mathrm{b}_{\mathrm{k}}{ }^{\mathrm{s}}$ are obtained from saturated cyclic stress- strain hysteresis curve (Jinghong and Xianghe, 1991).

Here, $b_{k}^{s}$ is the value of the coefficient of recall term as obtained from the saturation cycle of the hysteresis loop. In this model $\mathrm{C}_{\mathrm{k}}$, $b_{k}{ }^{s}$ are three sets of coefficients which are derived from three different segments of the saturated loop.

Next, $b_{k}^{s}=b_{k} \hat{b}\left(p_{s}\right)=\frac{b_{k}}{1+a p_{s}}$

Here, $\mathrm{p}_{\mathrm{s}}=$ saturated value of memory function

$\mathrm{a}=$ cyclic hardening rate. If $\mathrm{a}=0$, the effect of memory function on recall terms vanishes.

$b_{k}=$ The coefficient of recall term as obtained from the $1^{\text {st }}$ cycle of the hystersis loop.

The saturated value of memory function, $\mathrm{p}_{\mathrm{s}}$, is obtained from back stress Vs plastic strain curve of a saturated loop. This is as follows

$$
p_{s}=\frac{1}{\Delta \varepsilon_{p}} \int_{0}^{\Delta \varepsilon_{p}}\left|\xi\left(p_{s}\right)\right| d \bar{s}
$$

Here, $\xi$ is equal to the back stress of a saturated loop in uniaxial tension compression loading and $\Delta \varepsilon_{p}$ is the plastic strain range (i.e twice the plastic strain amplitude).

Since, 'a' and ' $\mathrm{b}_{\mathrm{k}}$ ' are related by an inequality, $a<\sqrt{\frac{2}{3}} \frac{b_{k}}{c_{k}}$, one has to proceed with a trial and error method. The first trial value of ' $a$ ' is obtained from equation (12). Knowing the values of $b_{k}{ }^{s}$ and $b_{k}$ and using the value of $p_{s}$ as given in equation (13) the value of ' $a$ ' can be determined from equation (12). The value of ' $a$ ' obtained from equation (12) is checked with the inequality,

$a<\sqrt{\frac{2}{3}} \frac{b_{k}}{c_{k}}$. Further adjustment in the value of 'a' may be required to obtain a better cyclic hardening rate.

The memory range, $\mathrm{S}_{0}$, is obtained as the accumulated plastic strain value of a loading branch in a saturated loop. Thus, $S_{0}=\int \dot{s} . d t$, over a loading branch in a saturated loop.

\section{Experiments and numerical calibration of kinematic hardening constants}

\subsection{Low cycle fatigue tests on tensile specimens:}

Uni-axial cyclic experiments are performed on $6 \mathrm{~mm}$ diameter specimens made of Sa333 Gr-6 carbon steel and SS-316 (Figure.1) under strain controlled (Figure.2) mode. A $100 \mathrm{kN}$ servo-hydraulic Universal testing machine is used. A $12.5 \mathrm{~mm}$ gauge extensometer is attached to the specimens to measure the strain during the tests. The strain-controlled tests are performed with the specimens for symmetric tension-compression strain cycles with the strain limits $\Delta \in= \pm 0.85 \%, \pm 1.0 \%, \pm 1.2 \%, \pm 1.6 \%$. The command for strain amplitude control are given in such a way so that the true strain amplitudes are maitained as mentioned above. The frequency of the tests are calculated for each strain amplitudes to maintain a strain rate of $.001 / \mathrm{s}$. Load, displacement and strains are recorded during the experiment and other parameters are calculated from these data. The stabilized hysteresis plot of $\sigma-$ $\in^{\mathrm{p}}$ for the strain range $\pm 1.0 \%$ is obtained from the test. This plot is used to calculate the Chaboche constants- $\left(\mathrm{C}_{1}, b_{1}^{s}\right),\left(\mathrm{C}_{2}, b_{2}^{s}\right)$, $\left(\mathrm{C}_{3}, b_{3}^{s}\right)$ in accordance with the procedure given by Bari and Hassan $(2000)$. Here, $b_{k}^{s}(\mathrm{k}=1,2,3)$ are the saturated values of the coefficients of recall term in kinematic hardening law (equation 5). The values of $b_{k}(\mathrm{k}=1,2,3)$ are also calculated from the $1^{\text {st }}$ cycle of the hysteresis loop. $p_{s}$ (saturated values of the memory function) is calculated from the saturated loop data of back stress vs plastic strain,Figure-3. (equation 13). The cyclic hardening rate ' $a$ ' is obtained from (equation 12). The constants obtained from the experiments are used in FE simulation.

\section{Finite element implementation of the model}

The FE simulation of the model is implemented on a round bar specimen (Figure.1) under strain controlled tension compression loading (Figure.2). An elasto plastic Finite Element code is developed in house with the material model discussed above. The fatigue specimen is modelled as axisymmetric loading with 8 noded element of mesh size as $0.2 \mathrm{~mm} \mathrm{X} 0.2 \mathrm{~mm}$. 
The calculation is done with the Von Mises yield function (equation 1) and Chobache's kinematic hardening rules modified with memory function (equation7). The stress strains are calculated at the center of the bar. The cyclic loading is plastic strain controlled. In Figure-4, back stress is plotted with axial plastic strain for saturated loop. In the first part of the diagram, the plastic strain amplitude is maintained at $\pm 0.805 \%$ (total strain amplitude is $\pm 1.0 \%$ ) with zero mean strain. It shows hardening and then it is saturated. The saturated value of back stress in tension and compression are $98.26 \mathrm{MPa}$ and $98.11 \mathrm{MPa}$ respectively. The response is symmetric with zero mean back stress.

After saturation, the strain level is extended to $3 \%$. The back stress achieved at this level is $129.95 \mathrm{MPa}$. Then it is cycled with same plastic strain amplitude of $\pm 0.805 \%$, i.e., between $1.39 \%$ to $3 \%$ with a mean strain of $2.195 \%$. The $2^{\text {nd }}$ part of Figure- 4 shows this cyclic behaviour. It shows softening and mean stress relaxation. The softening rate is slow.

Low cycle fatigue loops are simulated with finite element simulation. The first attempt is to simulate the saturated hyteresis loop. The saturated values of Chaboche's kinematic hardening coefficients are used. The material constant ' $\mathrm{a}$ ' and ' $\mathrm{S}_{0}$ ' are set to zero value. Thus, the memory function calculation is bypassed and its effect on kinematic hardening is deactivated .

The next attempt is to simulate cycle hardening in the transition cycle. This is to simulate the LCF loop from virgin state to saturation state. The values of material constants ' $\mathrm{a}$ ' and ' $\mathrm{S}_{0}$ ' are assigned. Thus, the memory function calculations are activated. The recall terms of Chaboche's kinematic hardening rules are calibrated with the memory function. The cyclic hardening is achieved.

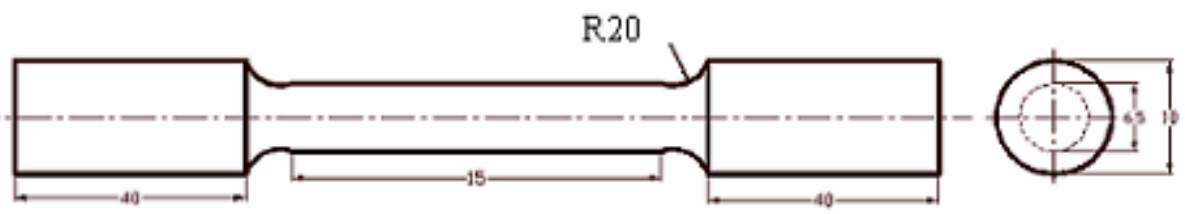

Figure 1. Uniaxial fatigue specimen

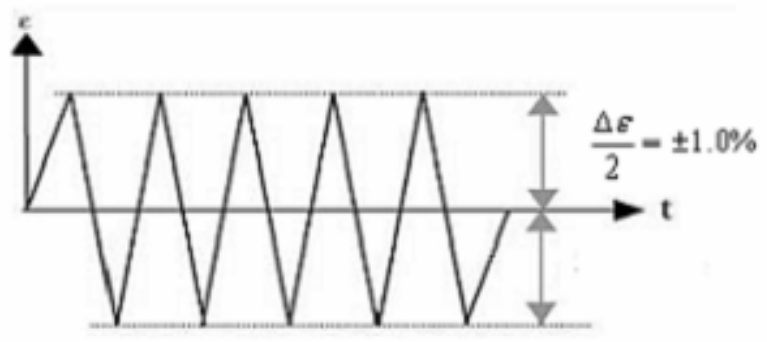

Figure 2. Loading history during strain controlled LCF test

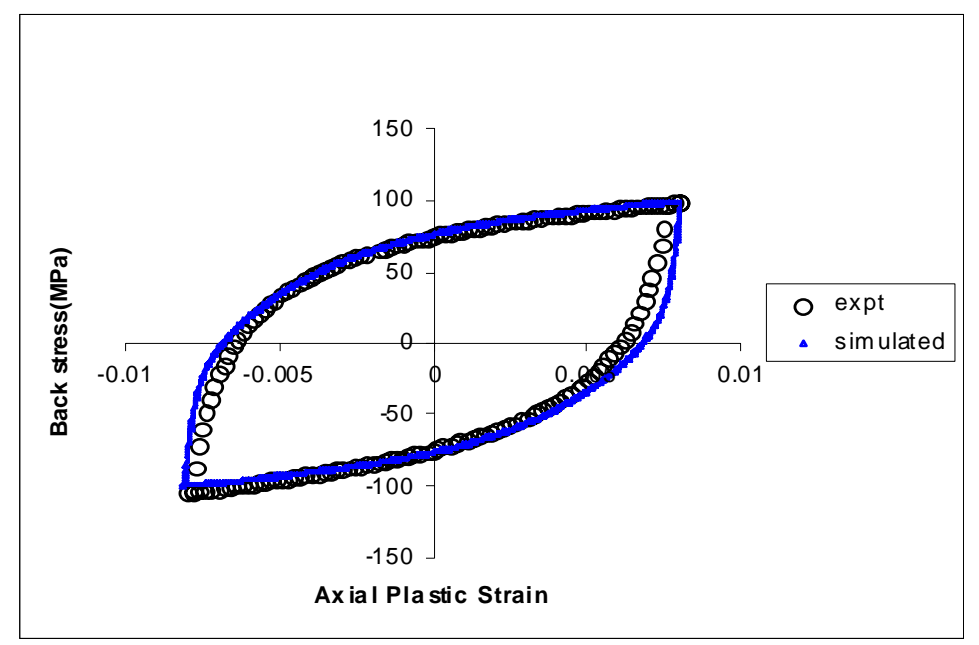

Figure 3. Back stress Vs plastic strain for strain amplitude $\pm 1.0 \%$ Material SA333 - Gr6 carbon steel 


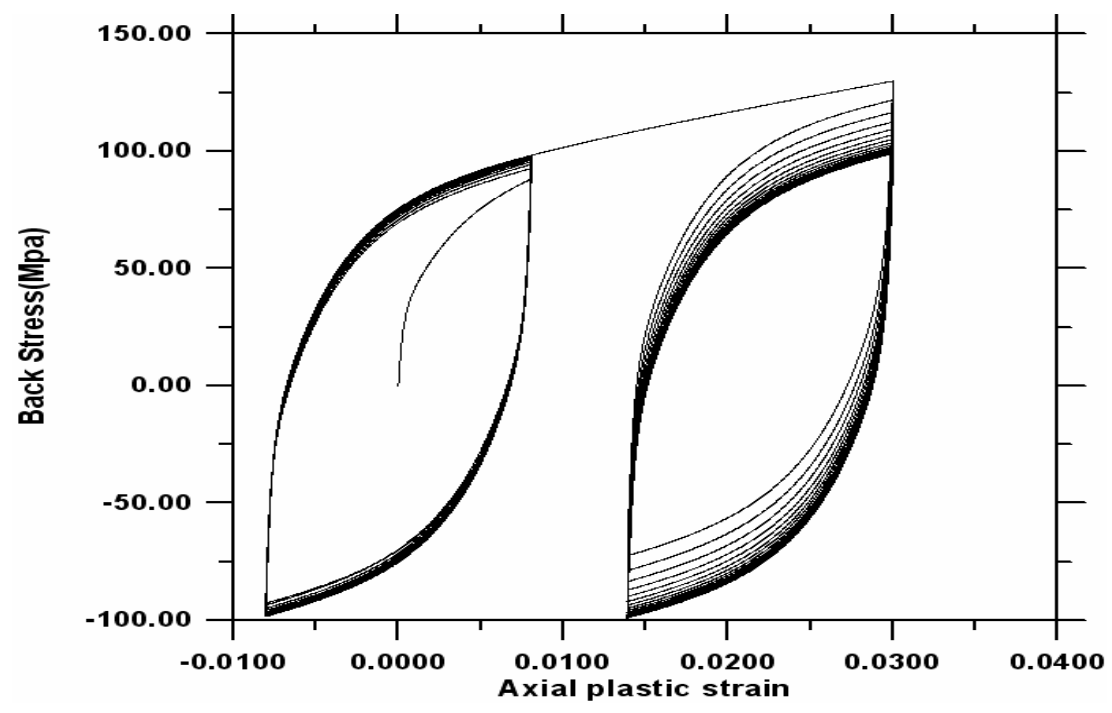

Figure 4. Mean stress relaxation for strain range $\pm 1.0 \%$ Material Sa 333 Gr-6 Carbon steel.

\section{Mechanical Properties of the Material SA333 Carbon Steel}

A. Tensile Properties

\begin{tabular}{|c|c|c|c|c|c|c|}
\hline \multirow{2}{*}{$\begin{array}{l}\text { Young's } \\
\text { Modulus (GPa) }\end{array}$} & \multirow[t]{2}{*}{ Poisson's Ratio } & \multirow[t]{2}{*}{$\sigma_{\mathrm{YP}}(\mathrm{MPa})$} & \multirow[t]{2}{*}{$\sigma_{\text {UTS }}(\mathrm{MPa})$} & \multirow[t]{2}{*}{$\%$ Elongation } & \multicolumn{2}{|c|}{$\sigma=\mathrm{K}\left(\varepsilon_{\mathrm{p}}\right)^{\mathrm{n}}$} \\
\hline & & & & & $\mathrm{K}$ & $\mathrm{n}$ \\
\hline 210 & 0.3 & 245 & 502 & 30 & 790 & 0.2432 \\
\hline
\end{tabular}

B. Kinematic Hardening Coefficients ( Saturated values)

$\mathrm{C}_{1}=110000 \mathrm{MPa}, \quad \mathrm{C}_{2}=14710 \mathrm{MPa}, \quad \mathrm{C}_{3}=1609 \mathrm{MPA}, \quad \gamma_{1}=3184, \gamma_{2}=271, \quad \gamma_{3}=0$

C. Kinematic Hardening Coefficients ( As obtained from $1^{\text {st }}$ cycle)

$\mathrm{C}_{1}=110000 \mathrm{MPa}, \quad \mathrm{C}_{2}=14710 \mathrm{MPa}, \quad \mathrm{C}_{3}=1609 \mathrm{MPA} \quad \gamma_{1}=4000.0, \quad \gamma_{2}=275, \quad \gamma_{3}=0$

D. Cyclic Hardening Coefficients

$\mathrm{S}_{0}=0.0548 \quad, \quad \mathrm{a}=0.00183585 \mathrm{MPa}^{-1}$

E. Calibration of Yield Stress with strain amplitude

\begin{tabular}{|l|l|}
\hline$\varepsilon_{\mathrm{P}}(\%)$ & $\left(\sigma_{\mathrm{YP}}\right)_{\mathrm{cyc}}(\mathrm{MPa})$ \\
\hline 1.37 & 285 \\
\hline 1.177 & 285 \\
\hline 1.0 & 258 \\
\hline 0.8 & 242 \\
\hline 0.6 & 238 \\
\hline
\end{tabular}




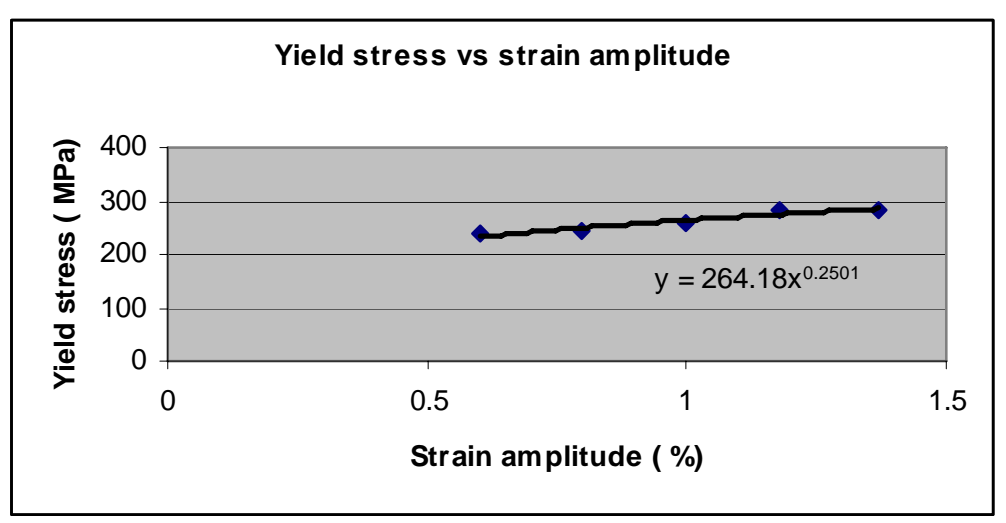

Figure 5. Yield stress from LCF loop at different strain amplitudes

\section{Results \& Discussion for Sa 333}

Figures. 6,7,8,9 show the FE simulated hysteresis loop $\sigma-\epsilon^{\mathrm{p}}$ for the saturated cycles. The results are compared with the experimental values. The Chaboche's constants are determined from the experimental saturated hysteresis loop for the strain amplitude $\pm 1.0 \%$. Same values of Chaboche's constants are used for other strain amplitudes. It is found that the simulated results match satisfactorily with the experimental results for the material Sa-333 carbon steel.

Figure 10 shows positive peak stress vs. cycles for the strain amplitude $\pm 0.85 \%$. Cyclic hardening is observed in the experiment. The cyclic hardening is getting saturated after 15 cycles. Also, the value of cyclic hardening for the material Sa333 is small. The first 50 cycles are simulated by FE computation. Although the simulated results do not match for initial 05 cycles the cyclic hardening rate matches after a few cycles. This shows the cyclic hardening rate coefficient 'a' ( equation(7)) is not properly calibrated with initial loop data. It is calibrated with data obtained after a few cycles.

Figure 11, Figure 12 and Figure-13 show similar results for the strain amplitude $\pm 1.0 \%, \pm 1.2 \%$. and $1.6 \%$.The similar observations are observed in those results also. The overall observation for cyclic hardening of the material SA333 is that the material shows initial hardening followed by little softening and then follows a steady small hardening rate. The simulated result matches with the steady hardening rate 


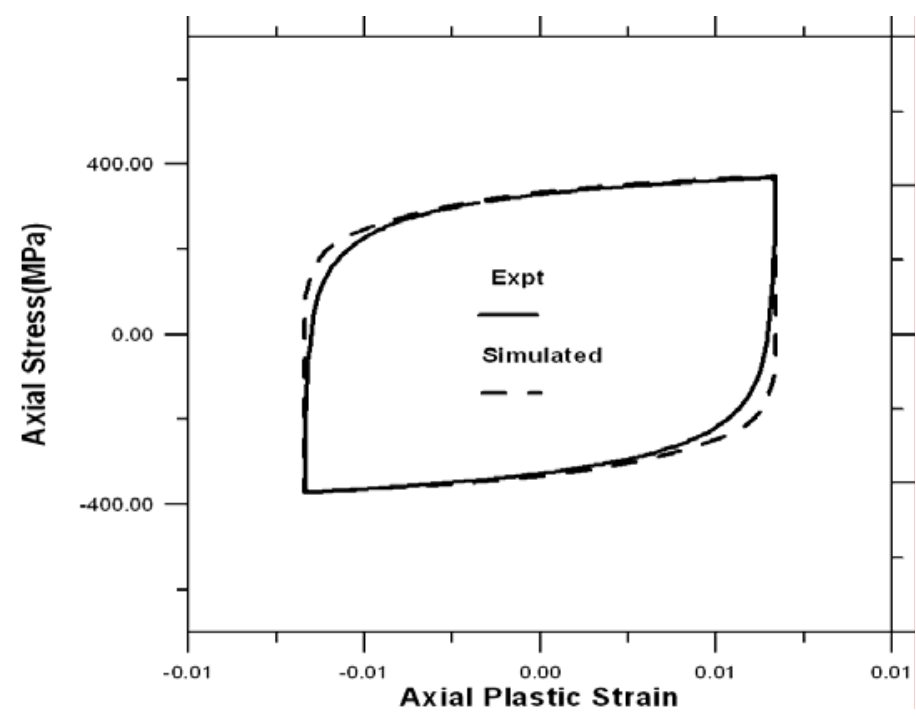

Figure 6. Simulation of saturated loop for strain range $\pm 0.85 \%$, material Sa 333 Gr-6 carbon steel.

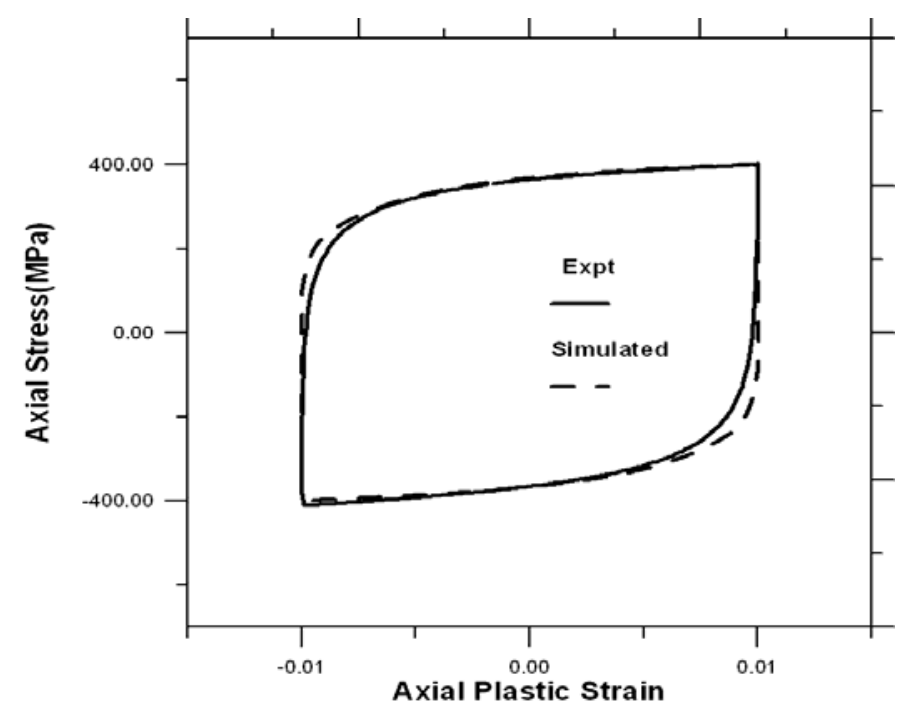

Figure 8. Simulation of saturated loop for strain range $\pm 1.2 \%$, material Sa 333 Gr-6 carbon steel.

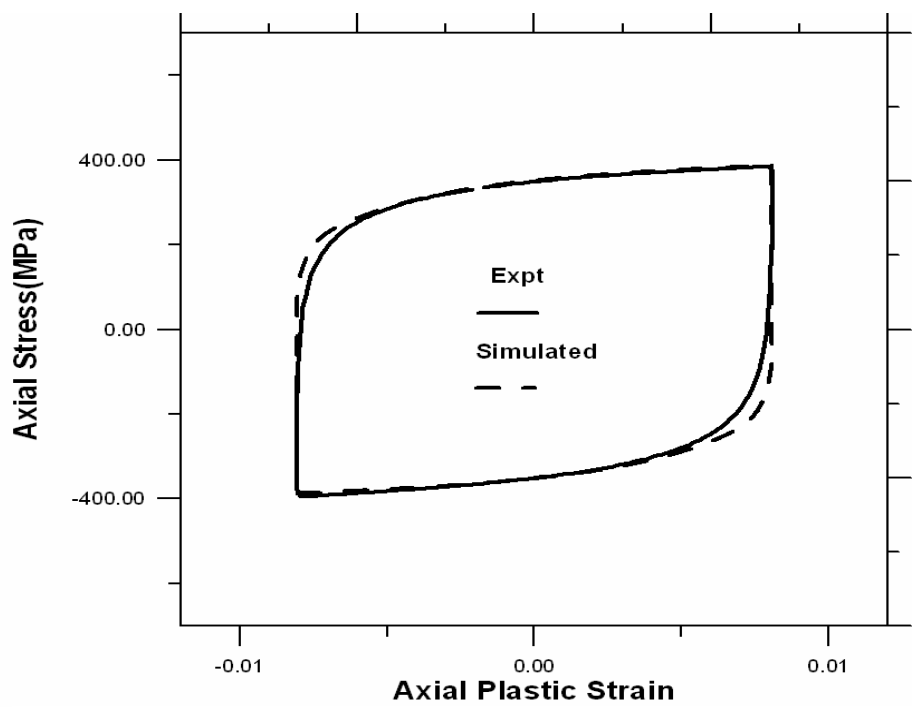

Figure 7. Simulation of saturated loop for strain range $\pm 1.0 \%$, material Sa 333 Gr-6 carbon steel.

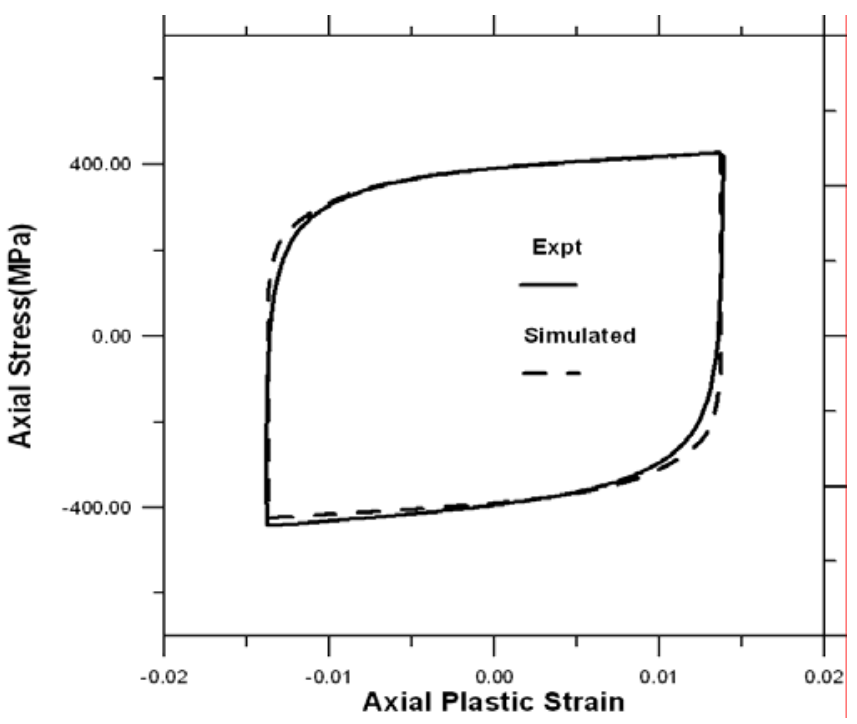

Figure 9. Simulation of saturated loop for strain range $\pm 1.6 \%$, material Sa 333 Gr-6 carbon steel. 

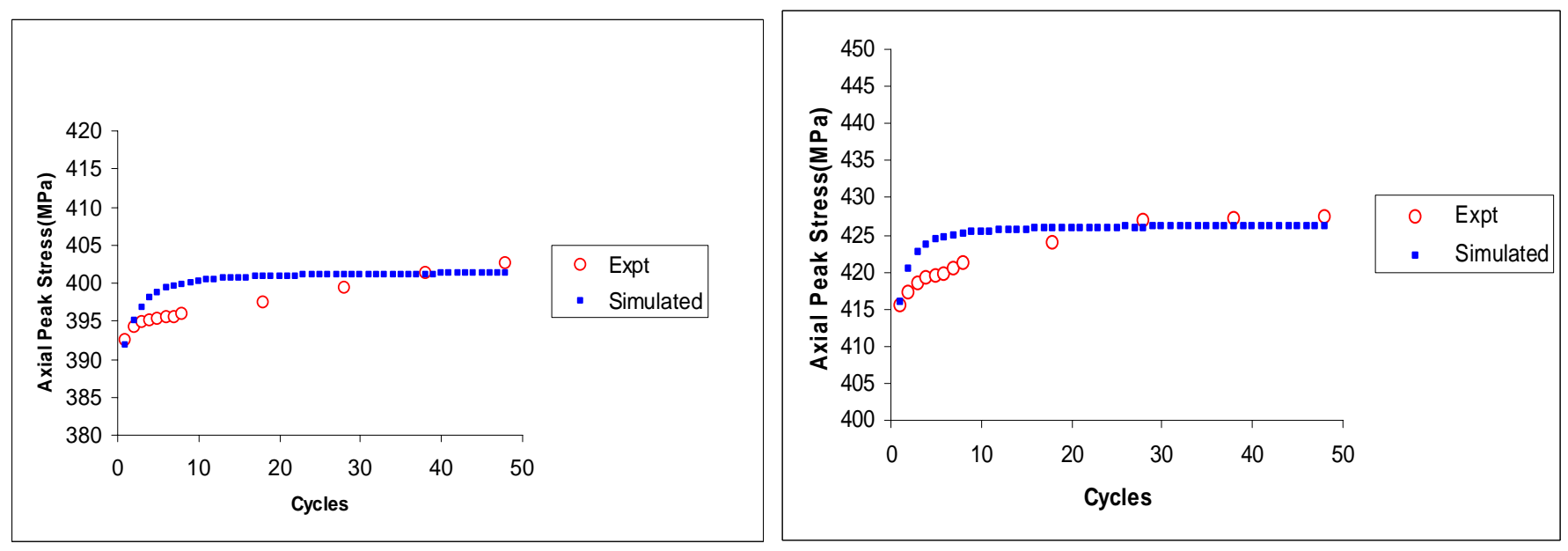

Figure 10. Peak Stress Vs Cycles for strain range $\pm 0.85 \%$ ( Mat- Sa333)

Figure 11. Peak stress Vs cycles for strain range $\pm 0.85 \%$ ( Mat- Sa333)

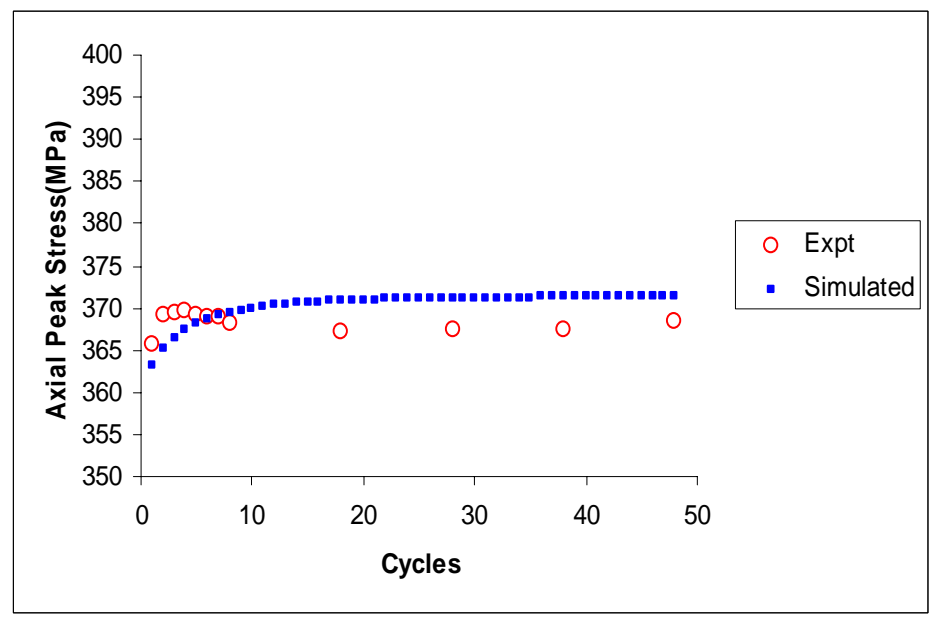

Figure 12. Peak Stress Vs Cycles for strain range $\pm 1.2 \%$

$$
\text { ( Mat- Sa333) }
$$

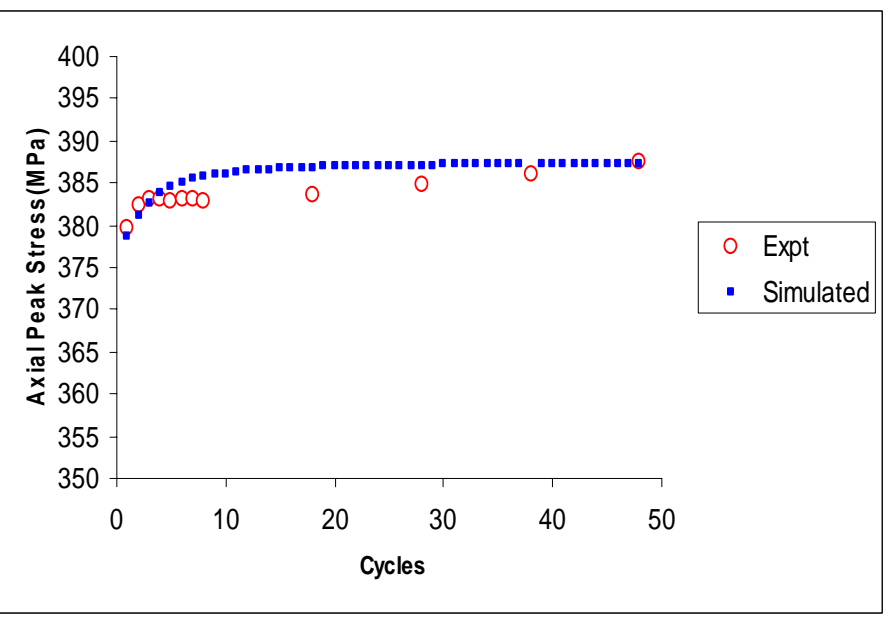

Figure 13. Peak stress Vs cycles for strainr range $\pm 1.6 \%$

( Mat- Sa333)

\section{Yield function of the material SS316}

For SS316, the yield function used is not a pure incompressible Von mises yield function.

Experimental results show higher peak stress in the compressive part of the loop than that in tensile part. To take care of this effect dilatation is introduced in the yield function.

Dialatation is introduced in the yield function in the following manner. Thus,

$\Phi=\frac{3}{2}\left(S_{i j}-X_{i j}\right)\left(S_{i j}-X_{i j}\right)-\sigma_{f}^{2}=0$ 
Here, flow stress, $\sigma_{f}=\sigma_{c}\left\{1-\beta\left(\frac{\sigma_{m}}{\sigma_{e q}}\right)\right\}$

Dilatation coefficient, $\beta=\frac{\dot{\varepsilon}_{v}^{p}}{\dot{\varepsilon}_{e q}^{p}}$

Here, $\dot{\varepsilon}_{v}^{p}$ is the volumetric plastic strain rate and $\dot{\varepsilon}_{e q}^{p}$ is the equivalent plastic strain rate.

Introduction of $\beta$ in yield function produces higher flow stress for negative $\sigma_{m}$ and reduces its value for positive $\sigma_{m}$ and hence strength differential effect introduced. The value of $\beta$ is calibrated to match the experimental LCF data.

\section{Mechanical Properties of SS316}

A. Tensile Properties

\begin{tabular}{|c|c|c|c|c|c|c|}
\hline \multirow{2}{*}{$\begin{array}{l}\text { Young's } \\
\text { Modulus (GPa) }\end{array}$} & \multirow[t]{2}{*}{ Poisson's Ratio } & \multirow[t]{2}{*}{$\sigma_{\mathrm{YP}}(\mathrm{MPa})$} & \multirow[t]{2}{*}{$\sigma_{\text {UTS }}(\mathrm{MPa})$} & \multirow[t]{2}{*}{$\%$ Elongation } & \multicolumn{2}{|c|}{$\sigma=\mathrm{K}\left(\varepsilon_{\mathrm{p}}\right)^{\mathrm{n}}$} \\
\hline & & & & & $\mathrm{K}$ & $\mathrm{n}$ \\
\hline 210 & 0.3 & 267 & 637 & 78 & 1150 & 0.286 \\
\hline
\end{tabular}

B. Kinematic Hardening Coefficients (Saturated values)

$\mathrm{C}_{1}=70000 \mathrm{MPa}, \quad \mathrm{C}_{2}=30000 \mathrm{MPa}, \quad \mathrm{C}_{3}=4000 \mathrm{MPA} \quad \gamma_{1}=1700, \quad \gamma_{2}=557, \quad \gamma_{3}=0$

C. Kinematic Hardening Coefficients (As obtained from $1^{\text {st }}$ cycle)

$\mathrm{C}_{1}=70000 \mathrm{MPa}, \quad \mathrm{C}_{2}=30000 \mathrm{MPa}, \quad \mathrm{C}_{3}=4000 \mathrm{MPA}, \quad \gamma_{1}=2200, \quad \gamma_{2}=800.0, \quad \gamma_{3}=0$

D. Cyclic Hardening Coefficients

$\mathrm{S}_{0}=0.04480 \quad, \mathrm{a}=0.003308 \mathrm{MPa}^{-1}$

E. Calibration of Yield Stress with strain amplitude

\begin{tabular}{|l|l|}
\hline$\varepsilon_{\mathrm{P}}(\%)$ & $\begin{array}{l}\left(\sigma_{\mathrm{YP}}\right)_{\mathrm{cyc}} \\
(\mathrm{MPa})\end{array}$ \\
\hline 0.81 & 215 \\
\hline 0.43 & 199 \\
\hline 0.33 & 196 \\
\hline
\end{tabular}

\section{Results and Discussions for SS316}

Figure 14 shows the comparison of simulated saturated hysteresis loop for a strain range $\pm 1.0 \%$ (plastic strain range $\pm 0.85 \%$ ) with the experimental one. It is found that $\beta=0.0$ gives higher peak stress in tension and $\beta=0.01$ gives higher peak stress in compression. For, $\beta=0.005$ the matching is satisfactory with respect to peak stresses in tension and compression. 


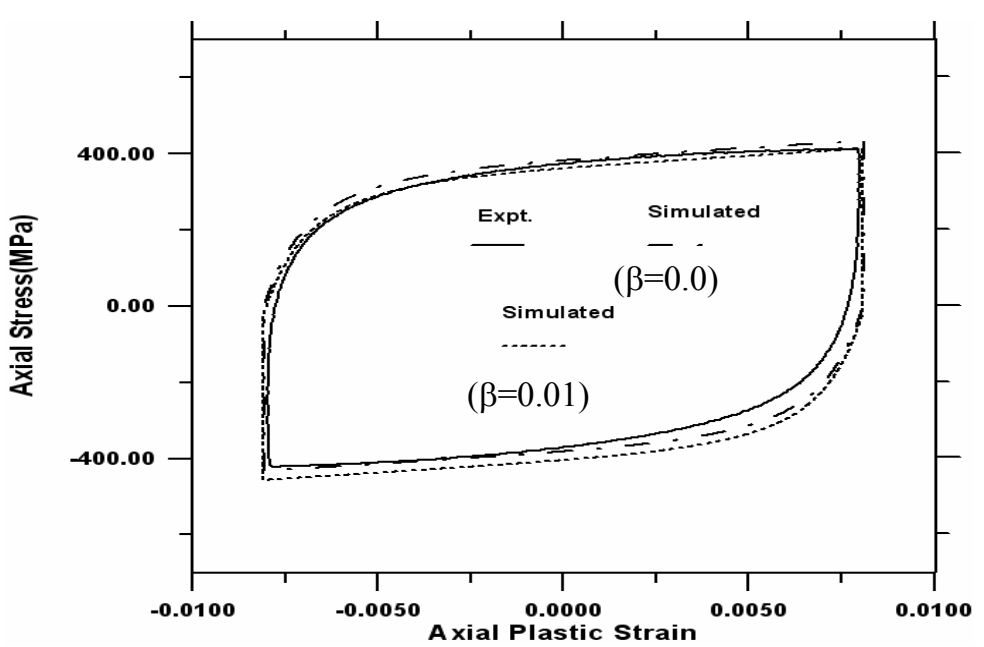

Figure 14. Saturated loop for strain range $\pm 1.0 \%$.

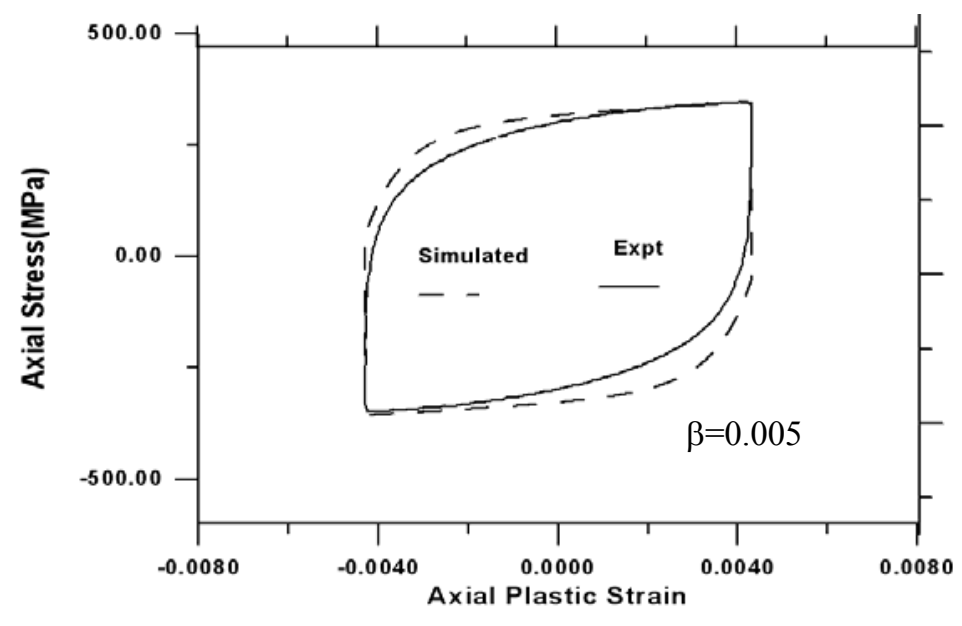

Figure 15. Saturate loop for srtain range $\pm 0.6 \%$

Material SS316

Material SS316

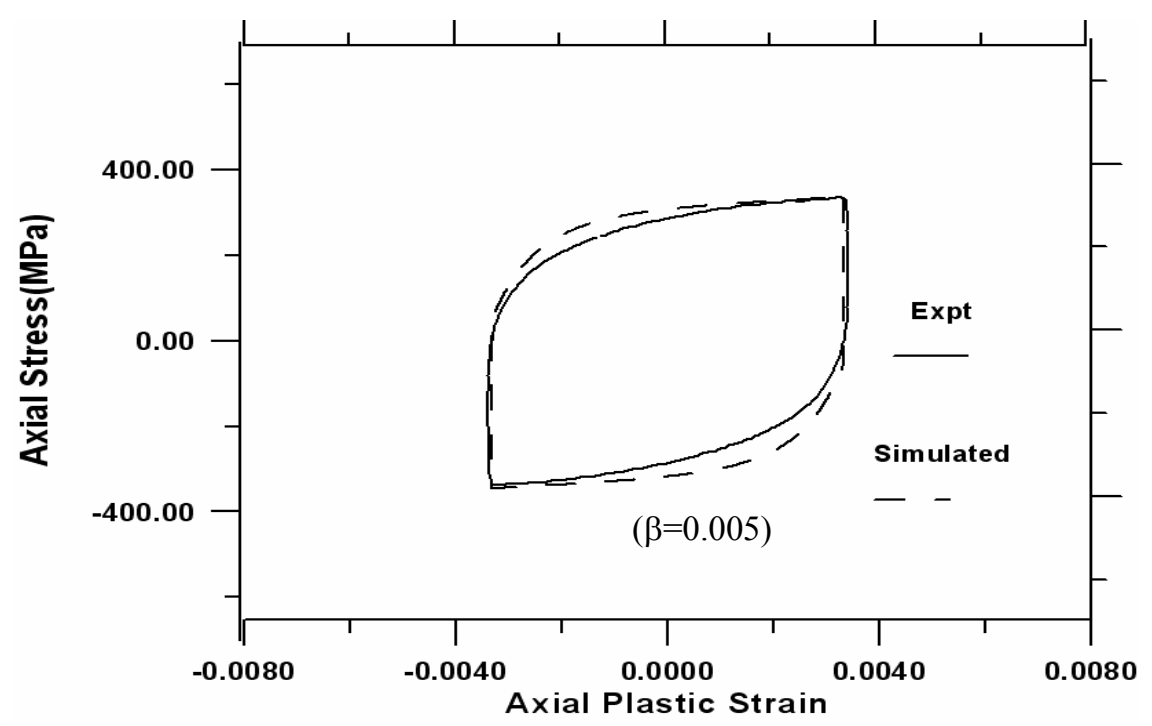

Figure 16. Saturated loop for strain range $\pm 0.5 \%$. Material SS316

The other curves ( Figure-15 and Figure 16) for strain ranges $\pm 0.6 \%$ and $\pm 0.5 \%$ are plotted with the same value of $\beta=0.005$. Overall, it is to say that the dilatation effect is not predominant for the material SS316 for lower strain range.

Figures 17, 18, 19 shows the peak stress Vs cycle curves for the material SS316. The first 20 cycles of the results are compared with the experimental values. It is observed that for small strain amplitudes ( Figure 15 and Figure 16) like $0.6 \%$ and $0.5 \%$ the effect of $\beta$ is negligible. For higher strain amplitude (Figure-19) of $1.0 \%$ the effect of $\beta$ is noticeable.

$\beta=0.01$ gives better matching with the experimental results. 


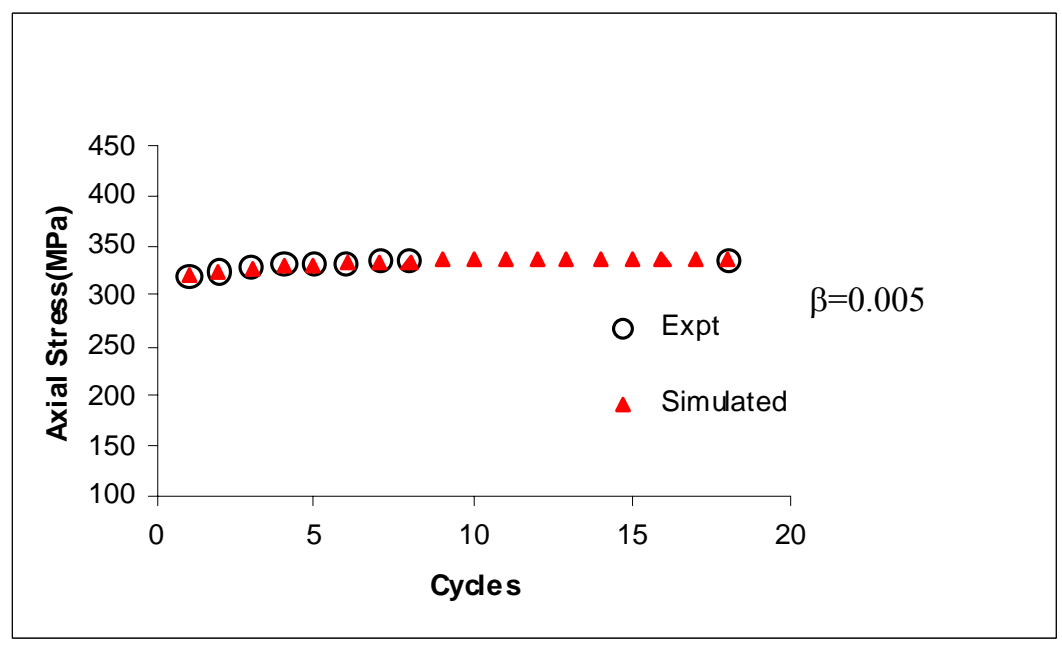

Figure 17. Peak stress Vs cycles for a strain range $\pm 0.5 \%$. Material SS316

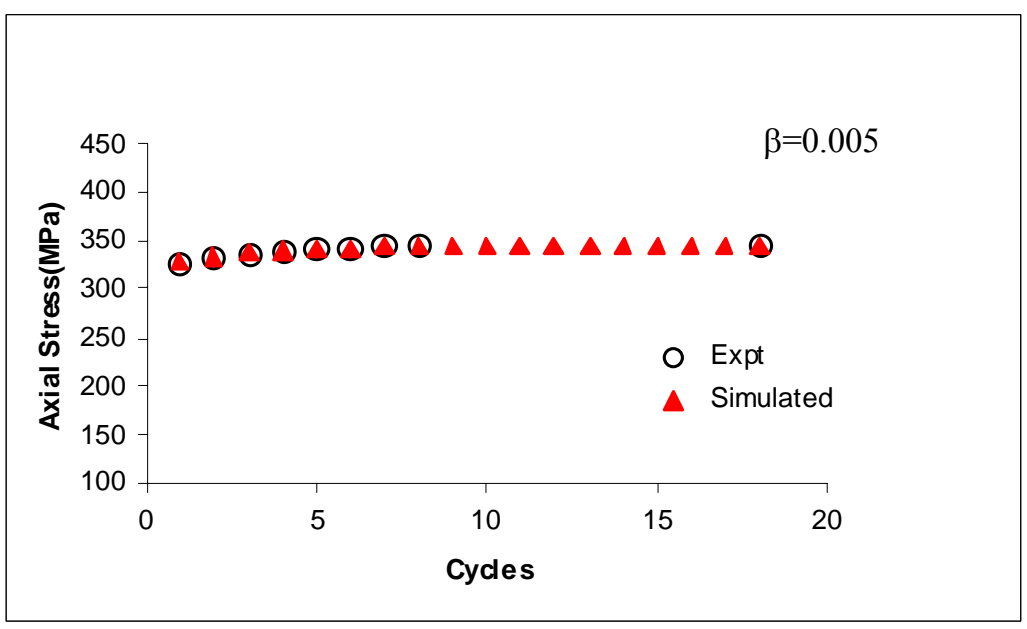

Figure 18. Peak stress Vs cycles for a strain range $\pm 0.6 \%$. Material SS316

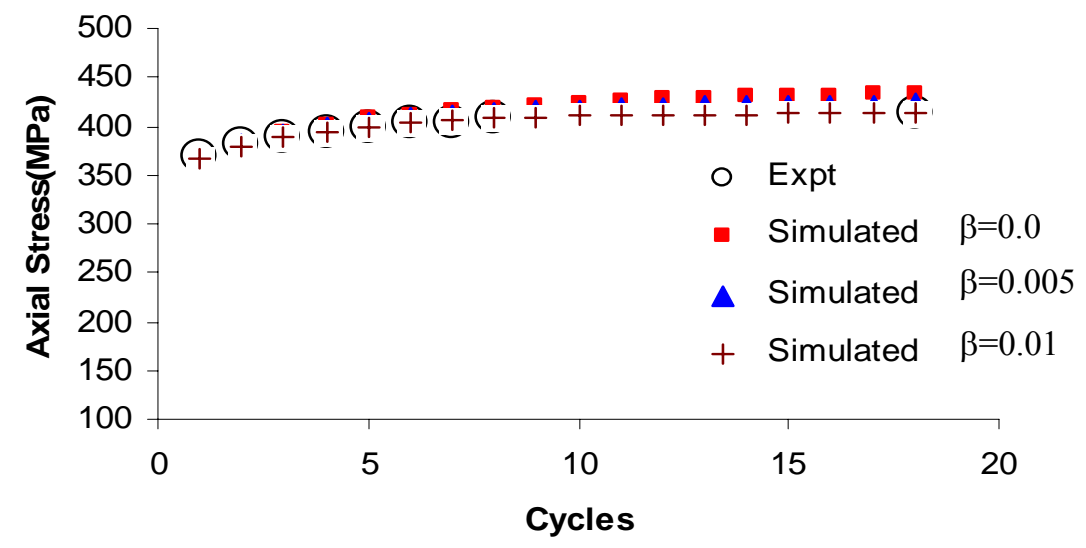

Figure 19. Peak stress Vs cycles for a strain range $\pm 1.0 \%$. Material SS316 


\section{Conclusion}

From the above results the following conclusions can be drawn.

1. The model presented in this article can simulate the transition state of the material Sa333 carbon steel in low cycle fatigue with some limitations. The model could not simulate the initial cyclic hardening followed by softening. But, the model simulates the steady hardening rate after few cycles. The complex behavior of the material Sa333 at the initial stage is due to large dynamic recovery which could not be addressed in this model.

2. Cyclic hardening rate is nicely simulated for the material SS316 stainless steel.

3. The dilation effect in the material SS316 is negligible for low strain amplitudes (below 1.0\%). The effect is noticeable at higher strain amplitudes.

4. Overall, the saturated LCF loops are simulated satisfactorily for both the materials.

\section{Nomenclature}

a cyclic hardening rate

$b_{k}, c_{k}$ parameter used in the Chaboche model

E Young's modulus

s accumulated plastic strain

$S_{i j} \quad$ deviatoric stress tensor

$S \quad$ magnitude of incremental plastic strain

$\mathrm{p}(\mathrm{s}) \quad$ memory function

$\sigma_{i j} \quad$ stress tensor

$S_{0} \quad$ memory range

$\sigma_{c} \quad$ yield stress

$\mathrm{X}$ back stress tensor

$\varepsilon_{i j} \quad$ total strain tensor

$\dot{X}$ incremental back stress tensor

$\varepsilon_{i j}^{e} \quad$ elastic strain tensor

$X_{k} \quad$ kth component of incremental back stress tensor

$\varepsilon_{i j}^{e} \quad$ incremental elastic strain tensor

$\varepsilon_{i j}^{p} \quad$ plastic strain tensor

$\varepsilon_{i j}^{p} \quad$ incremental plastic strain tensor

$v \quad$ Poisson's ratio

$\dot{\varepsilon}_{v}^{p} \quad$ volumetric plastic strain rate

$\dot{\varepsilon}_{e q}^{p} \quad$ equivalent plastic strain rate

$\Delta \varepsilon \quad$ total strain range

$\lambda$ plastic multiplier

$\Phi \quad$ yield function

$\beta \quad$ dilatation coefficient

\section{References}

Armstrong P.J, Frederick C.O. 1966. A mathematical representation of the multiaxial Bauschinger effect. CEGB; Report No $\mathrm{RD} / \mathrm{B} / \mathrm{N} 731$.

Benallal A, LeFallo P, Marquis D. 1989. An experimental investigation of cyclic hardening of 316 stainless steel and 2024 aluminum alloy under multiaxial loading. Nucl. Engg. Des, Vol. 114, pp. 345.

Bari S, Hassan T. 2000, Anatomy to coupled constitutive models for ratcheting simulation. Int J. Plasticity; Vol.16, pp. 381-409.

Chaboche J.L, Dang Van, Cordier G. 1979. Modelization of the strain memory effect on cyclic hardening of 316 stainless steel. Paper L $11 / 3 ; 5^{\text {th }}$ SMIRT, Berlin. 
Chaboche J.L, Rousselier G. 1983. On the plasticity and viscoplasticity constitutive equations. JL. Pressure Vessel Tech; Vol.105, pp. 153-164.

Chaboche J.L. 1986. Time independent constitutive theories for cyclic plastic. Int. JL. Plasticity, Vol. 2, No. 2, pp. 149-188.

Ellyin F, Xia Z. 1989, A rate independent constitutive model for transient non proportional loading. JL. Mech. Phys. Solids, Vol.37, No. 1, pp. 71-91.

Haupt P, Korzen M, Ch. Tsakmakis. 1987. ZAMM; 67:T193.

Haupt P, Kamlah M. 1995. Representation of cyclic hardening and softening properties using continuous variables. Int. JL. Plasticity, Vol. 11, pp. 267-291.

Jinghong F, Xianghe P.1991. A psysically based constitutive description for non proportional cyclic plasticity. JL. Engg. Mat. Tech, Vol. 113, pp. 254-262.

Mahnken Rolf. 2001. Strength difference in tension and compression and pressure dependence of yielding. Comp. Meth. Appl. Mech. Engg, Vol.190, pp. 5057-5080.

Ohno N. A , 1982. Constitutive model of cyclic plasticity with a non hardening strain region. ASME, JL. Appl. Mech, Vol-49, pp. 721-727.

Ohno N, Kachi Y. A. 1985. Constitutive model of cyclic plasticity for nonlinear hardening material. JL. Appl. Mech, Vol. 53, pp. 395-403.

Prager. W 1956. A new method of analyzing stresses and strains in work hardening plastic solids. ASME J. Appl.Mech Vol. 23 , pp. 493-496.

Spitzig W.A, Sober R.J, Richmond O. 1975. Pressure dependence of yielding and associated volume expansion in tempered martensite. Acta Metall, Vol. 23, No. 7, pp. 885-893.

\section{Biographical notes}

Dr. Sankar Dhar is a Professor of Mechanical Engineering, Jadavpur University (India). He is engaged in teaching and research activities since the last 25 years. His field of specialization is Plasticity and Ductile fracture. Dr. Dhar has a lot of experience in supervising several projects from BARC and DRDO. He worked in CEA, France for two years. He has published several papers in various national, international conferences and journals. He has guided 3 students for their Ph.D. work.

Dr. Sanjib Acharyya is working as a Reader in Mechanical Engineering Department, Jadavpur University, India. He is engaged in teaching and research activities since the last 18 years. His field of specializations are Ductile fracture and Material Characteristion and Design Optimisation. Dr. Acharyya has been supervising several projects from BARC and DRDO. He has published several papers in various national, international conferences and journals. At present he is guiding 4 students for their Ph.D. work.

Jagabandhu Shit is a Lecturer in Raigangue Politechnic College, West Bengal. He is doing his Ph.D in the area of LCF and ratcheting in Mechanical Engineering Department, Jadavpur University. He has completed his PG from Mechanical Engineering Department Jadavpur University.

Received December 2009

Accepted March 2010

Final acceptance in revised form May 2010 